\title{
Dynamics of in-process control with non-contact air gauges
}

\author{
Mirosław Rucki ${ }^{1}$ \\ ${ }^{1}$ Faculty of Mechanical Engineering, Kazimierz Pulaski University of Technology and Humanities in Radom, Poland, \\ e-mail: m.rucki@uthrad.pl
}

\begin{tabular}{l} 
Article Info \\
\hline Article history: \\
Received November 1, 2020 \\
Revised December 10, 2020 \\
Accepted December 12, 2020
\end{tabular}

\section{Keywords:}

Dimensional measurement; Air gauging; Time constant; Dynamic error.

\begin{abstract}
Dynamic properties of the air gauges performing in-process measurement are of the great importance because of dynamic error affecting the measurement results. The paper presents the analysis of the air gauges dynamics and some practical recommendation. The investigations proved the dependence of the time constants on the actually measured back-pressure. In practical solutions of in-process control, the air gauge must work in conditions of falling backpressure since with the material removal dimensions of the machined workpiece go down. Thus, in the area of the smallest values of back-pressure within the measuring range, the time constant value is the largest. Worsening of the air gauge dynamic properties at the end stage of the machining must be considered when the dynamic characteristics of the projected air gauge are calculated.
\end{abstract}

Copyright (C) 2020 Regional Association for Security and crisis management and European centre for operational research. All rights reserved.

\section{Corresponding Author:}

Mirosław Rucki,

Faculty of Mechanical Engineering, Kazimierz Pulaski University of Technology and Humanities in

Radom, Poland.

Email: m.rucki@uthrad.pl

\section{Introduction}

In-process measurements ensure high productquality through real-time quality monitoring, reduce manufacturing costs and increase productivity (Berger et al., 2015). Automated quality control is very important aspect of a manufacturing process (Milo et al., 2015), and the measurement accuracy is particularly important in the new manufacturing era called Smart Manufacturing, Intelligent Factory or Industry 4.0 (Moroni \& Petro, 2018). Metrology becomes part of the Cyber-Physical Manufacturing Systems (CPMS) and involves big data analytics (Majstorovic et al., 2018) as well as Internet of Things (Majstorovic et al., 2019). To handle metrological data in multistage manufacturing processes, intelligent systems based on neural networks are applied (Papananias et al. 2019). The intelligent embedded systems have a potential to contribute to more efficient manufacturing and more manageable technological processes (Tomov \& Dimitrov, 2019).

Scientists and engineers appreciate many merits of the pneumatic dimensional measurement, among others the simple construction of gauge, cheap maintenance, easy regulation of metrological properties, non-contact measurement, self-cleaning of the measured surface with the outflowing air (in open jet devices) and so on (Curtis \& Farago, 2014). In 1960ths and 1970ths, the air gauging was important part of the metrological sciences with numerous publications around the World (Lotze, 1968; Pogorelov, 1971; Zalmanzon, 1971). After some decline in the development, air gauges regained new attention (Schuetz, 2008; Koenig 2017), especially in the context of the in-process control (Menzies \& Koshy, 2009; Vacharanukul \& Mekid, 2005; Koehn 2015; Rao 2017). In particular, researchers looked for the ways of improvement of static and dynamic characteristics of the air gauges (Jermak 2010; Cellary \& Jermak 1997; Jakubowicz \& Derezynski 2017), proposed some modifications of the nozzle geometry (Jakubowicz 2020), and evaluated new models of their 
work (Bokov 2011; Jermak 2017). However, dynamical properties of an air gauge are considered rather poor, e.g. in documentation Millimar (2005) its setting time is declared as 0.3-0.5 s.

Application of piezoresistive transducers and small chambers was desribed by Rucki et al. (2010), it reduced the time constant and setting time down to few milliseconds (Rucki \& Jermak, 2012). As a result, the air gauges became suitable in the applications of high dynamics. This study is dedicated to the accurate determination of the time constant, which is crucial in in-process measurement with air gauges.

\section{Typical In-Process Back-Pressure Air Gauging}

Typical air gauging measurement during the machining is widely known (Yandayan \& Burdekin, 1997). In short, the back-pressure air gauge is monitoring the diameter of machined detail, and after the set value is reached, the spool valve controls the parameter of machining or stops the process. Of course, in case of electronic signal gained from the piezoresistive pressure transducer, it could be processed by computer and used in more sophisticated digital control system as well as in the industrial process database (Sanchez et al., 2020).

It should be noted, however, that when machining shafts, the work of the air gauge starts at larger diameters of the workpiece which provide smaller slot $s$ between the detail surface and measuring nozzle. Subsequently, during the machining process, the material is removed and the slot becomes wider, and corresponding backpressure falls down. In some range $z_{p}$, the dependence of the back-pressure $p_{k}$ on the slot width $s$ is proportional. The completion of the machining process would correspond with slot $s$ close to the end of this proportional area when the back-pressure $p_{k}$ is the smallest.

Similarly, in case of machining of inner cylinder surface, the in-process measurement starts with smaller slots and ends with larger ones, since the machining is causing the removal of the material. E.g. during the honing process, the inner diameter of cylinder grows larger, and the measured slot width s grows larger, too. The slot between the measuring nozzle and the inner cylinder surface is illustrated in Figure 1 . The diameters of inlet and measuring nozzles are denoted $d_{w}$ and $d_{p}$, respectively. The area between them is called the measuring chamber, where the back-pressure $p k$ dependent on slot width $s$ is measured. The stabilized feeding pressure of the air gauge is denoted $p_{z}$, and its value, along with nozzles geometry, determines the metrological characteristics of the air gauge (Derezynski \& Jakubowicz, 2016).

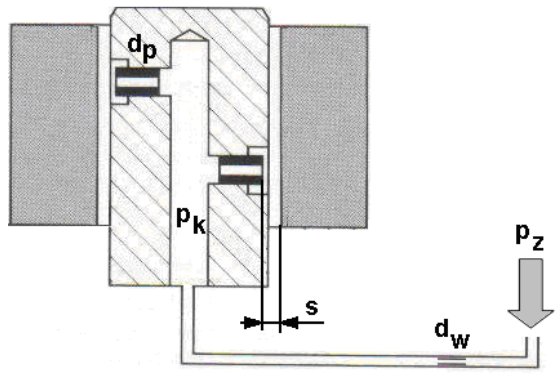

Figure 1. Slot width $s$ in measurement of inner diameters

In the example of the Figure 1, it is seen that the volume of measuring chamber between restriction $d_{w}$ and measuring nozzle $d_{p}$ is relatively big. It naturally affects the dynamical properties of the air gauge, according to the formula (Zelczak, 2002):

$$
T=\frac{v_{k} \rho_{0} \tau_{0} R g}{p_{0}}\left(\frac{\partial G_{2}}{\partial w_{2 s r}} \frac{\partial w_{1 s r}}{\partial p_{k}}\right)-\left(\frac{\partial G_{1}}{\partial w_{1 s r}} \frac{\partial w_{1 s r}}{\partial p_{k}}\right)
$$

where: $v_{k}$ - volume of measuring chamber, $\rho_{0}$ - mass density, $\tau_{0}-$ temperature, $R$ - gas constant, $g-$ acceleration of gravity, $G_{1}$ and $G_{2}$ - kinetic energy of the air stream in the inlet and measuring nozzles, $w_{l s r}$ and $w_{2 s r}$ - mean velocity of the air in the inlet and measuring nozzles.

In order to reduce the time constant, smaller volumes of measuring chambers should be applied. It was proposed, e.g. in (Jermak \& Rucki, 2016) where three measuring nozzles were not joined in one measuring chamber, but worked independently with three small measuring chambers. The measuring head was placed on the flexible rod, and the data analysis was based on the original algorithm, which calculated the roundness of the measured detail from three independent signals. This device was designed for quality control performed 
offline, after a part is produced (Gao et al., 2014), when the material is no longer removed. However, in most in-process applications, the air gauges produce the control signal when the proper amount of the material is removed and the gauge works in the end area of its measuring range $z_{p}$. This area corresponds with larger slot width $s$ and smaller back-pressure $p_{k}$.

\section{Materials and Methods}

Measurement of the air gauge dynamic characteristics was performed with the specially arranged laboratory set described in details by Jakubowicz, Rucki \& Siemiatkowski (2019). It consisted of mechanical sine input in form of eccentric shaft, electrical motor with settable rotational speed to control the input frequency, and the electronic signal processing and computational unit. Three main sources of uncertainty were considered, namely, mechanical generation of the sine input $s(\omega t)$, pressure transducer indications $p_{k}$, and further signal processing with estimations, smoothening, averaging, interpolation and similar procedures. Overall system uncertainty in determination of time constant $T$ for coverage factor $k=2$, which corresponded with approximately $95 \%$ level of confidence, was calculated as $U_{95}=0.001 \mathrm{~s}$ (Jakubowicz, Rucki \& Babic, 2019).

Conficurations of air gauges used in experiments are described in Table 1. The examined air gauge with $d_{p}=1.200$ combined with $d_{w}=1.200 \mathrm{~mm}$ (denoted D2 in Table 1$)$ and $d_{w}=0.625 \mathrm{~mm}$ (denoted D5 in Table 1) provided the multiplication of $K=0.15 \mathrm{kPa} / \mu \mathrm{m}$ and $K=0.88 \mathrm{kPa} / \mu \mathrm{m}$ respectively. Therefore, the measuring ranges $z_{p D 2}$ and $z_{p D 5}$ correspond with different ranges of back-pressure, as it is seen in Figure 2 . Both combinations were joined with the measuring chambers of reduced volume $v_{k} 0.402$ and $3.921 \mathrm{~cm}^{3}$.

Table 1. Examined configurations of air gauges

\begin{tabular}{cccccc}
\hline No. & $d_{p}[\mathrm{~mm}]$ & $d_{w}[\mathrm{~mm}]$ & $K[\mathrm{kPa} / \mu \mathrm{m}]$ & $v_{k}\left[\mathrm{~cm}^{3}\right]$ & $p_{k}$ range $[\mathrm{kPa}]$ \\
\hline 2D2 & 1.200 & 1.200 & 0.15 & 0.402 & $105-140$ \\
9D2 & 1.200 & 1.200 & 0.15 & 3.921 & $105-140$ \\
2D5 & 1.200 & 0.625 & 0.88 & 0.402 & $70-115$ \\
9D5 & 1.200 & 0.625 & 0.88 & 3.921 & $70-115$ \\
\hline
\end{tabular}

a)

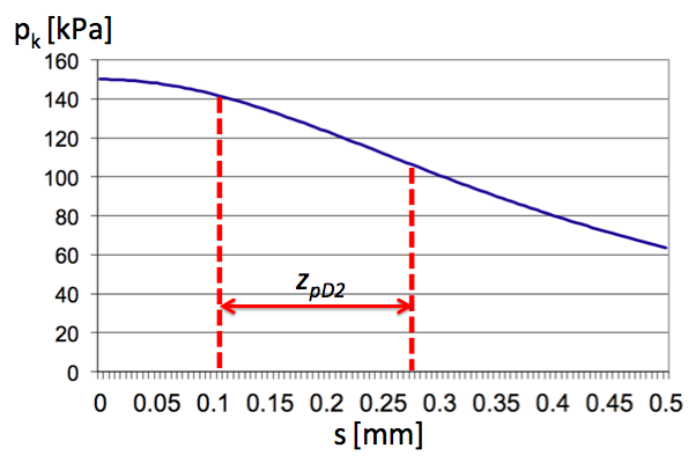

b)

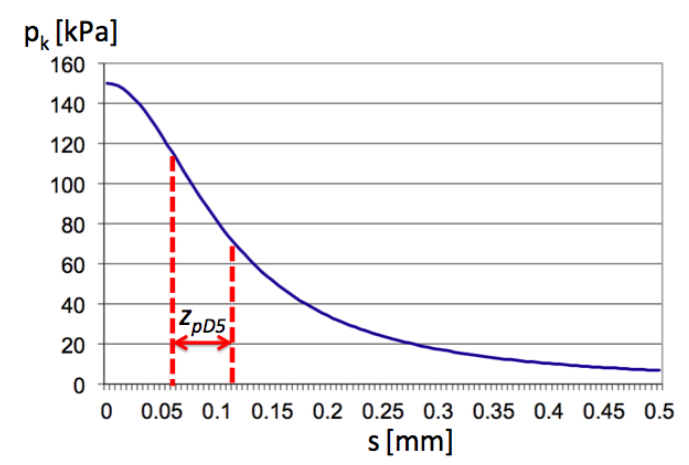

Figure 2. Static characteristics of the examined air gauges: (a) with $d_{p}=1.200$ and $d_{w}=1.200 \mathrm{~mm}$;

(b) with $d_{p}=1.200$ and $d_{w}=0.625 \mathrm{~mm}$.

In previous studies, it was demonstrated that under certain conditions, dynamics of the small chamber air gauges could be described in terms of the first-order system (Rucki \& Jermak, 2012). Thus, it is possible to calculate the phase shift as following (Figliola \& Beasley, 2006):

$\varphi(\omega)=-\operatorname{arctg}(\omega T)$

and

$\varphi(\omega)=-\operatorname{arctg} \frac{Q(\omega)}{P(\omega)}$

Hence, the sine response of the system could be described by formula: 
$y(t)=C e^{-t / T}+\frac{K A}{\sqrt{1+(\omega T)^{2}}} \sin [\omega t-\varphi(\omega)]$

In Figure 3a, there are presented graphs of the theoretical phase shift calculated according to the formula (3) and the experimental one. It should be noted that for the examined case the 5\% dynamic error is reached for the frequency $f_{0.95}=2.6 \mathrm{~Hz}$ shown in the amplitude-frequency characteristics in Figure $3 \mathrm{~b}$. This value corresponds with $\omega=16.3$ and $\omega T=0.33$. In that extend, the working frequency below $2.6 \mathrm{~Hz}$ is the object of interest. The analysis proved that in the point of $\omega T=0.33$, the difference between the experiment and firstorder dynamic system was smaller than $8 \%$.

a)

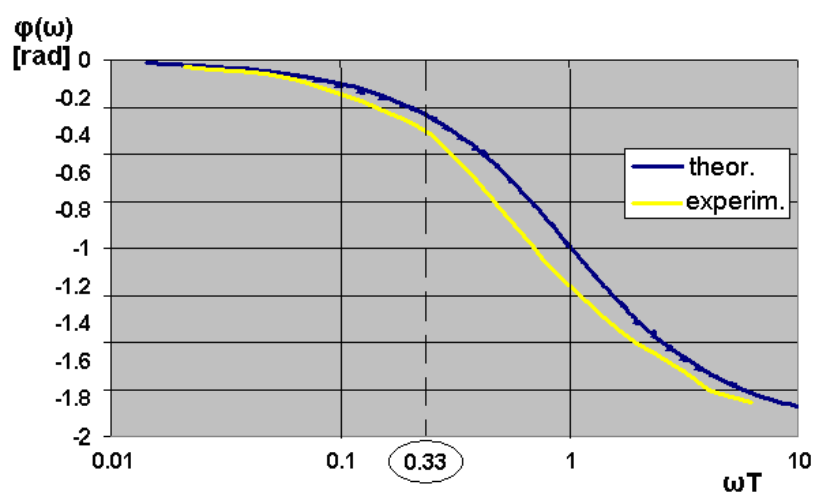

b)

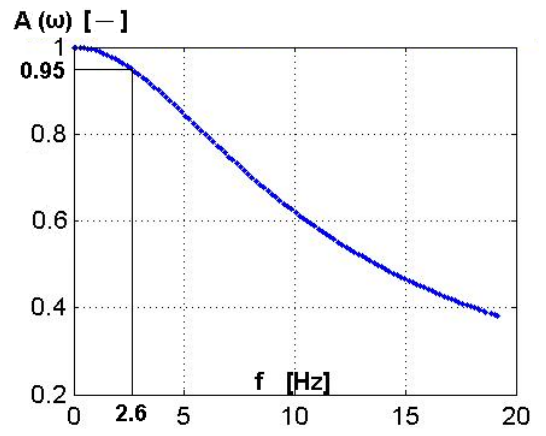

Figure 3. Dynamic characteristics of the examined air gauge with $d_{p}=1.200$ and $d_{w}=0.625 \mathrm{~mm}$ :

(a) Phase characteristics; (b) Amplitude-frequency characteristics.

\section{Pressure-Dependent Time Constant}

It was found that for the vessels with pressured air, in the process of emptying there is dependence of the time constant on the actual pressure in the vessel (Koscielny \& Wozniak, 1995):

$T=\frac{1}{M}\left(1-\frac{p_{a}}{p_{0}}\right)$

where: $p_{a}, p_{0}$ - atmospheric pressure and pressure in the vessel, respectively; $M$ - factor of proportionality.

When such pressure dependence $T=f\left(p_{k}\right)$ is considered, the approximation with exponential function is much closer to the experimental results and fits to them with the error smaller than $3 \%$. Figure 4 a presents the graph of function $T=f\left(p_{k}\right)$, and Figure $4 \mathrm{~b}$ shows how new approximation fits to the experimental results.

a)

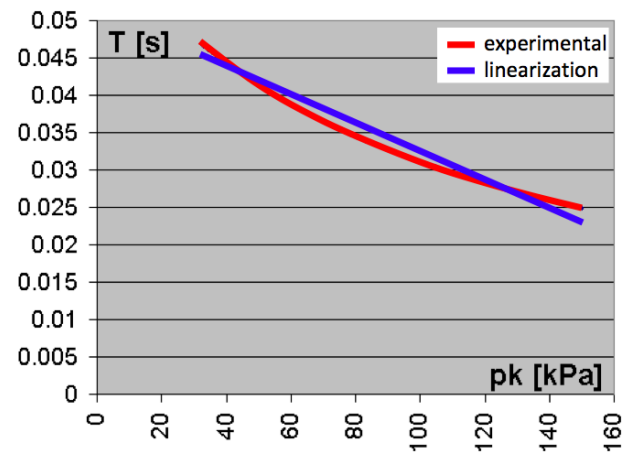

b)

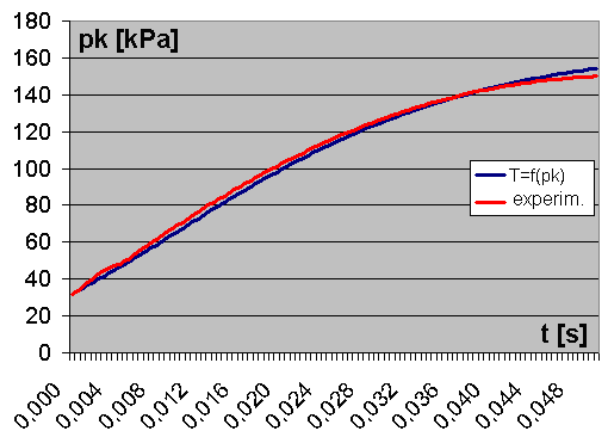

Figure 4. Dependence of the time constant on the air gauge with $d_{p}=1.200$ and $d_{w}=0.625 \mathrm{~mm}$ : (a) Graph of the function $T=f\left(p_{k}\right)$; (b) Calculated step response considering the function $T=f\left(p_{k}\right)$ in comparison to the experimental data. 
For the practical reasons in industrial applications, the function $T=f\left(p_{k}\right)$ can be linearized. Using the Least Square method, as it is shown in the Figure 9a with blue line, the linearization error is ca. 7\%. However, exact analysis of the laboratory measurement results provided interesting observations specified below.

The functions of $T=f\left(p_{k}\right)$ for each examined air gauge configuration, appeared to be dependent on measuring chamber volume $v_{k}$ with some distinguishable similarity. Figure 5 presents the graphs of time constants dependent on the back-pressure and slot width for the air gauges specified in the Table 1 . It is seen that smaller volumes of measuring chambers and smaller multiplication $K$ of the air gauge provide smaller sensitivity of time constant $T$ to the actual back-pressure $p_{k}$.

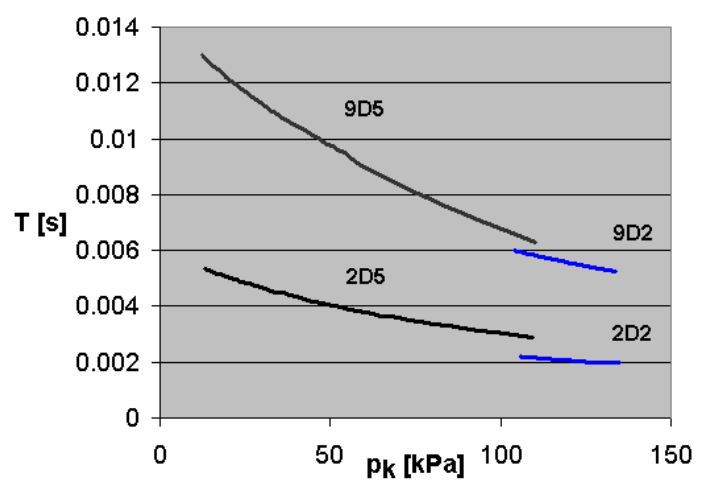

Figure 5. Examples of the $T=f\left(p_{k}\right)$ functions for the examined air gauges configurations.

From the Figure 5, following correlations can be derived.

As it could be expected, the time constatnt $T$ is highly dependent on the measuring chamber volume and can be reduced ca. $50 \%$ when $v_{k}$ is reduced from ca. $4.0 \mathrm{~cm}^{3}$ down to $0.4 \mathrm{~cm}^{3}$. However, for the same volume $v_{k}$, decrease of the inner nozzle diameter $d_{w}$ from 1.2 down to $0.6 \mathrm{~mm}$, and subsequent increase of the miltiplication $K$ from 0.15 up to $0.88 \mathrm{kPa} / \mu \mathrm{m}$ has substantial effect on the time constant. This effect is twofold. On the one hand it depends on actual back-pressue $p_{k}$, so that larger measured slot $s$ within the same measuring range is measured with longer time constant $T$. On the other hand, the entire curve of $T(s)$ is placed a little higher for smaller $d_{w}$ providing higher time constant for the same $s$ and respective back-pressure $p_{k}$. In the examined cases, at $p_{k}=110 \mathrm{kPa}$, black curves are placed little higher than the blue ones.

The most important observation is related to the curvature of $T(s)$ for different configurations of air gauges. In general, larger volumes $v_{k}$ provided higher dependancy of time constant on the back-pressure. In case of 2D2 configuration, the differences in time constant $T$ for different $p_{k}$ values are small and could be neglected. However, in case of 9D5 such a difference is more than 100\%, from 6 up to $13 \mathrm{~ms}$. In all cases, however, declination of the $T(s)$ curve is increasing with the increase of $s$ and respective $p_{k}$.

Figure 6 shows schematic in-process control of the grinding with air gauges. After each stroke, the initial slot width $s$ is enlarged with Depth Of Cut (DOC) value, causing respective back-pressure drop $\Delta p_{k}=p_{k 1}-p_{k 2}$. When the control system registeres the final value $p_{k f}$, the grinding is finished.

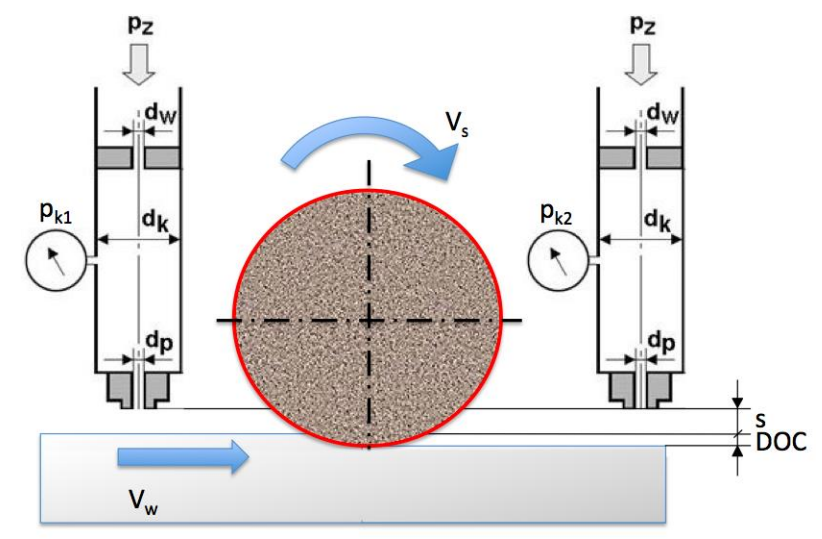

Figure 6. Scheme of the in-process measurement of the grinded plate 
However, with larger slot width corresponds smaller back-pressure, which take place at the end of the machining process. In that very moment, the dynamics of the air gauge is the worst. Increased time constant provides the maximal possible dynamic error. Control device could identify the proper dimensional signal $p_{k f}$ too late, and the process could go too far, removing too much material.

\section{Conclusions}

The theoretical analysis and experimental researches on dynamics of air gauges led to the conclusion that its time constant could be reduced down to several milliseconds. That could be reached when the pressure transducer has very small time constant, e.g. piezoresistive one with $T=0.1 \mathrm{~ms}$. Next, the measuring chamber volume should be reduced down to few $\mathrm{cm}^{3}$ or even less than $1 \mathrm{~cm}^{3}$. Under such a conditions, the air gauge reach time constant of several milliseconds and could be treated as a first-order dynamic system.

Obviously, smaller measuring chambers provide smaller time constants. It was proved, however, that the time constant of the high-multiplication air gauge is larger than that of low-multiplication ones. Moreover, the time constant depends on the actual value of back-pressure. In-situ measurement during the technological process requires high dynamics of the measurement device especially in the final stage of the machining. For the air gauges, that means the range of the lowest back-pressure, where the time constants are longest. The largest dynamic error in the most responsible moment of measurement could cause that the system would generate the control signal too late.

Thus, in-process application of air gauge demands more thorough analysis of time constant in the conditions of falling back-pressure, for its smallest values. It is important especially in case of high-multiplication air gauges with larger volumes of measuring chambers. Small volumes, e.g. less than $1 \mathrm{~cm}^{3}$, and low multiplication of the air gauge provide the time constant almost insensitive to the back-pressure changes.

Acknowledgement: The paper is based on the researches performed in the Laboratory of Pneumatic Devices in Division of Metrology and Measurement Systems at Poznan University of Metrology, Poland. Author wishes to thank all the staff members for cooperation and friendliness.

\section{References}

Berger, D., Brabandt, D., \& Lanza, G. (2015). Conception of a mobile climate simulation chamber for the investigation of the influences of harsh shop floor conditions on in-process measurement systems in machine tools. Measurement, 74, 233-237.

Bokov, V.B. (2011). Pneumatic gauge steady-state modelling by theoretical and empirical methods. Measurement, 44, 303-311.

Cellary, A., \& Jermak, Cz.J. (1997). Dynamics of One-Cascade Pneumatic Sensor for the Length Measuring. Optoelectronic and Electronic Sensors, 3054, 36-39.

Curtis, M.A., \& Farago, F.T. (2014). Handbook of Dimensional Measurement. ( $5^{\text {th }}$ ed.). New York: Industrial Press Inc.

Derezynski, J., \& Jakubowicz, M. (2016). Verification tests of the air gauges metrological characteristics. Mechanik, 3, 196-199. (in Polish)

Figliola, R.S., \& Beasley D.E. (2011). Theory and Design for Mechanical Measurements. Clemson University: John Wiley \& Sons Inc.

Gao, R.D., Tang, X., Gordon, G., \& Kazmer, D.O. (2014). Online product qualty monitoring through inprocess measurement. CIRP Annals - Manufacturing Technology, 63(1), 493-496.

Jakubowicz, M. (2020). Accuracy of roundness assessment using air gauge with the slot-shaped measuring nozzle. Measurement, 155, Article 107558.

Jakubowicz, M., \& Derezynski, J. (2017). The measuring position designed to determine the metrological properties of air gauges. Advances in Science and Technology Researches, 11(4), 198-205.

Jakubowicz, M., Rucki, M., \& Babic, M. (2019). Uncertainty of Sine Input Calibration Apparatus for the Air Gauges. In M. Diering, M. Wieczorowski, \& Ch.A. Brown (eds.), Advances in Manufacturing II, Volume 5 - Metrology and Measurement Systems, Lecture Notes in Mechanical Engineering (pp. 82-94). Cham: Springer, 2019, s. 82-94. https://doi.org/10.1007/978-3-030-18682-1_7

Jakubowicz, M., Rucki, M., \& Siemiątkowski, Z. (2019). Computer approximation of air gauge dynamic characteristics using the sine input test rig. MATEC Web of Conferences, 252, Article 02002, https://doi.org/10.1051/matecconf/201925202002

Jermak, Cz.J, \& Rucki, M. (2016). Dynamics of the non-contact roundness measurement with air gages. Acta mechanica et automatica, 10(3), 227-232. 
Jermak, Cz.J. (2010). Methods of Shaping the Metrological Characteristics of Air Gages. Strojniški vestnik Journal of Mechanical Engineering, 56(6), 385-390.

Jermak, Cz.J. (2017). Discussion on flow-through phenomena in the air gauge cascade. Acta Mechanica et Automatica, 11(1), 38-46.

Koehn, Ch. (2014). In-process air gaging. Quality Magazine, 53(5), 22-23.

Koenig, B. (2017). Marriage of convenience. Manufacturing engineering, 158(3), 59-63.

Koscielny, W., \& Wozniak, C. (1995). Experimental evaluation of flow models in pneumatic resistances. Proceedings of $9^{\text {th }}$ National Polish Conference PNEUMA-1995, Kielce, Poland, pp. 83-92.

Lotze, W. (1968). Critical assessment and contributions to the development of pneumatic length measurement technology. Habilitation, TU Dresden. (in German)

Majstorovic, V., Stojadinovic, S., Jakovljevic, Z., Zivkovic, S., Djurdjanovic, D., Kostic, J., \& Gligorijevic, N. (2018). Cyber-Physical Manufacturing Metrology Model (CPM3) - Big Data Analytics Issue. Procedia CIRP, 72, 503-508.

Majstorovic, V., Zivkovic, S., Djurdjanovic, D., Sabbagh, R., Kvrgic, V., \& Gligorijevic, N. (2019). Building of Internet of Things Model for Cyber-Physical Manufacturing Metrology Model (CPM3). Procedia CIRP 81, 862-867.

Menzies, I., \& Koshy, P. (2009). In-process detection of surface porosity in machined castings. International Journal of Machine Tools \& Manufacture, 49(6), 530-535.

Millimar. Length Metrology Components and Systems. (2005) Catalogue on Dimensional Metrology, Mahr GmbH, Esslingen - Göttingen.

Milo, M.W., Roan, M., \& Harris, B. (2015). A new statistical approach to automated quality control in manufacturing processes. Journal of Manufacturing Systems, 36, 159-167.

Moroni, G., \& Petro, S. (2018). Managing uncertainty in the new manufacturing era. Procedia CIRP, 75, 1-2.

Papananias, M., McLeay, Th.E., Mahfouf, M., \& Kadirkamanathan, V. (2019). An Intelligent Metrology Informatics System based on Neural Networks for Multistage Manufacturing Processes. Procedia CIRP, 82, 444-449.

Pogorelov, V.I. (1971). Gasodynamic calculations of the air gauges. Moscow: Mashinostroenie. (in Russian)

Rao, P.S., Jain, P.K., \& Dwivedi, D.K. (2017). Optimization of key process parameters on electro chemical honing $(\mathrm{ECH})$ of external cylindrical surfaces of titanium alloy Ti 6Al 4V. Materials Today: Proceedings, 4(2 Part A), 2279-2289.

Rucki, M., Barisic, B., \& Varga, G. (2010). Air Gauges as a Part of the Dimensional Inspection Systems. Measurement, 43(1), 83-91.

Rucki, M., Jermak, Cz.J. (2012). Dynamic Properties of Small Chamber Air Gages. Journal of Dynamic Systems, Measurement, and Control, 134(1), Article 011001.

Sanchez, M., Exposito, E., \& Aguilar, J. (2020). Autonomic computing in manufacturing process coordination in Industry 4.0 context. Journal of Industrial Information Integration, 19, Article 100159.

Schuetz, G. (2008). Air Gaging Gets Better with Age. Quality Magazine, 3, 28-32.

Tomov, P., \& Dimitrov, L. (2019). The role of digital information models for horizontal and vertical interaction in intelligent production. Facta Universitatis Series: Mechanical Engineering, 17(3), 397-404.

Vacharanukul, K., \& Mekid, S. (2005). In-Process dimensional inspection sensors. Measurement, 38(3), 204218.

Yandayan, T., \& Burdekin, M. (1997). In-process dimensional measurement and control of workpiece accuracy. International Journal of Machine Tools and Manufacture, 37(10), 1423-1439.

Załmanzon, W. A. (1971). Teoria elementów stosowanych w technice strumieniowej. Warszawa: WNT. (in Polish)

Zelczak, A. (2002). Pneumatic Length Measurements. Warsaw: WKL. (in Polish) 